\title{
Risk and regulation in research
}

One major theme of this issue of the Journal of Medical Ethics is research ethics and its relationship to risk. Unusually, this issue discusses research involving animals as well as human participants. In their editorial, building on two papers in this issue, ${ }^{12}$ Yeates and Reed of the RSPCA point out that the public, major funders of animal research, are calling for greater transparency (see page 504).

The risk of increased transparency is that it will inhibit research, and therefore limit the potential benefits. Yates and Reed conclude, "That is a reason for improvements, not for a lack of complete transparency." I would add that better communication of the necessity, the reasonableness of risk and the potential benefits would also help to balance the public's perception of such trials.

Having sat on several research ethics committees scrutinising research involving human participants, I have found the balance between protecting participants and facilitating good research is a fine one. I have argued that a pernicious tendency for a legalistic approach to ethical concepts such as informed consent has replaced a genuine consideration of the balance between individual risks and benefits, ${ }^{3}$ and that the primary duty of ethics committees is to ensure that risk is reasonable. ${ }^{4}{ }^{5} \mathrm{In}$ this issue, Bromwich and Rid use risk to apply a gradation of consent to allow for a less burdensome process of obtaining consent where risks are low, even when the participant is not likely to receive a benefit themselves from the trial (see page 521, Editor's choice). They argue the informed consent process should be adapted to risk, complexity, controversy and potentially other factors. Therefore in moderate and high risk trials, the simple provision of large amounts of information is not sufficient for Bromwich and Rid: the researcher must verify that the participant has understood that information.

The legalistic approach can mean participants are left to sign off on acres of small print. The researcher has done their duty and has the signed form to prove it. As Bromwich and Rid point out, such an approach is invalid. Of course, Bromwich and Rid's proposal places additional burden on the ethics committee to ensure trials are appropriately labelled low, moderate or high risk. But that should be part of ethics committees' remit to scrutinise and minimizing harm.

In a similar vein, Hunter looks at prerecruitment of trial participants, often run by private companies, and seeks to balance the efficiency of pre-recruitment in streamlining research, with the risks of this practice which is currently unregulated (see page 557). He proposes a short-term solution: that ethics committees review prerecruitment literature as part of their scrutiny of trials. This provides a safety net whilst not adding a significant additional burden.

Hunter, however, seeks in the longer term, further regulation of such companies. This is an interesting question. Assuming that valid consent is obtained prior to participating in a trial, is the act of signing up with a company to receive news about these trials itself an ethical issue? For Hunter, it appears the major issue lies with the advertising used by these companies which highlights the financial compensation and the human benefits of research, framing participation as 'heroic'. Again this is a difficult balance. Hunter is concerned that participants will enter trials for the wrong reasons. Yet we do pay research participants, and their participation is necessary for research to proceed.

I am not as conservative as Hunter. Indeed, I have argued that research participants should be paid more for their efforts ${ }^{6}$ in recognition of the risks that they take. As for research benefits, trials are assessed for their likely benefit as part of the approval process. Positive results can't be guaranteed; but it is a managed risk, and one that is explained to participants prior to trial entry. (As an aside, if progress is made on the logging of all clinical trials, there would be a better case that all results would add to the sum of knowledge: Alltrials.net runs an ongoing campaign in this area). I don't wish to trivialise the risks involved, and the Northwick Park case and others is a stark reminder that the risks are real. But again, there is a delicate value judgement about the reasonableness of all the relevant risks that ethics committees must make.

One of the fascinating aspects of medical ethics is its interaction with new scientific developments. One recent example has been the discovery that some patients previously thought to be in a vegetative state are in fact able to respond (in some form) to researchers. Relatives of these patients are of course anxious to know this information, and in this issue Graham et al. (who include in their team a number of the researchers at the cutting edge of this research), tease out the ethical issues around informing families, especially in the light of remaining uncertainty over interpreting results (see page 534). Given the range of abilities detected in such patients, including: "the capacity for sustained attention (required to maintain focus), language comprehension (required to understand instructions), response selection (required to switch between alternative tasks or conditions) and working memory (required to remember which task to perform when instructed)", and the ability to answer yes or no answers, I imagine further issues will arise around consent: could such a patient have the capacity to provide valid consent on their own behalf? Moreover, could they provide valid refusal of life-prolonging medical treatment? As in the evaluation of risk in research, complex ethical judgements await in this field about the worth of life with disorders of consciousness ${ }^{7}$ as well interpreting any wishes the patient may be able to express. Families will need to be involved in such judgements, which makes the ethics even more complex.

\section{REFERENCES}

1 Levin LH, Reppy WA. Reforming the politics of animal research. J Med Ethics 2015;41:564-7.

2 Goodman J, Chandna A, Roe K. Trends in animal use at US research facilities. J Med Ethics 2015;41:568-70.

3 Savulescu J. 2014. One Success in Research Ethics. Practical Ethics in the News. http://blog.practicalethics. ox.ac.uk/2014/11/one-success-in-research-ethics/ (accessed 8 Jun 2015).

4 Savulescu J, Hope T. 'Ethics of Research'. In Skorupski J, ed. The Routledge Companion to Ethics. Abingdon: Routledge, 2010:781-95.

5 Savulescu J. 'Two Deaths and Two Lessons: Is it Time to Review the Structure and Function of Research Ethics Committees?' J Med Ethics 2002:28;1-2.

6 Savulescu J. 'Taking the Plunge'. New Scientist 2001:2280:50.

7 Savulescu J, Kahane G. 'Brain-Damage and the Moral Significance of Consciousness'. Journal of Medicine and Philosophy 2009;34:6-26. 